\title{
Adaptive finite element analysis with quadrilateral elements using a new $h$-refinement strategy
}

\author{
C S KRISHNAMOORTHY* and S MUKHERJEE \\ Department of Civil Engineering, Indian Institute of Technology, \\ Madras 600036, India \\ e-mail: moorthy@civil,iitm.ernet.in
}

\begin{abstract}
The theory and mathematical bases of $a$-posteriori error estimates are explained. It is shown that the Medial Axis of a body can be used to decompose it into a set of mutually non-overlapping quadrilateral and triangular primitives. A mesh generation scheme used to generate quadrilaterals inside these primitives is also presented together with its relevant implementation aspects. A new $h$-refinement strategy based on weighted average energy norm and enhanced by strain energy density ratios is proposed and two typical problems are solved to demonstrate its efficiency over the conventional refinement strategy in the relative improvement of global asymptotic convergence.
\end{abstract}

Keywords. Adaptive finite element analysis; quadrilateral elements; $h$ refinement strategy.

\section{Introduction}

The reliability and accuracy of finite element analysis (FEA) has always been a point of contention, especially in applications where the precision of the solution is critical to the analyst. In general, it can be stated that although FEA is the most widely used tool for the solution of a large class of engineering problems characterized by PDEs (Partial Differential Equations), the accuracy of the solution may always be questioned. From a global point of view, this inaccuracy may be attributed to the modelling drawbacks of FEA since it is practically impossible to characterize the infinite number of degrees of freedom of a real physical system by a discrete numerical model. This modelling deficiency usually results in a lower bound of the solution which is manifested by stiffening in structural mechanics problems.

In most real life engineering analysis problems, classical solutions are almost never available since the problem domain is usually non-regular. In such cases, the FE solution is the only benchmark that can be taken as a reference and thus it is imperative that it is reliable and accurate.

${ }^{*}$ For correspondence 
In this aspect, one more feature of FEA should also be addressed - viz. automation of the procedure. The reason for this is two-fold: first, the automation of the FE procedure implies a lesser amount of man-machine interaction which reduces the chance of human errors and, second, as will be shown later, error estimation procedures perform best in automatic unstructured mesh generating environments - so, in a way automation enhances the reliability of the error estimation procedure. To address the problems of accuracy estimates and reliability of the FE solution, a closer insight to typical FE errors is thus required. A study of the derivation of the error estimates using variational bases is also necessary to gain further insight into the relationship these estimates share with the standard FE process.

\section{Errors in FEA}

FE solution errors may be broadly classified into three major groups depending upon their source of generation. It may be stated that the various sources of errors are inherent in the modelling of the continuum problem into a discrete set of equations, rounding off and truncation due to limited representation and operation of floating point variables in the computer itself and in the overstiffening effect of the structural system in general. From these viewpoints, FE solution errors may be classified as given below

\section{- Mathematical modelling errors}

These occur due to the fact that no mathematical model can fully satisfy all the characteristics of a physical model. Thus, such errors are introduced at the very onset of the formulation of the PDEs.

\section{- Discretization errors}

In a discretized FE model there are a finite number of degrees of freedom which are used to model a continuum system which has practically an infinite number of degrees of freedom. This overstiffens the system and produces discretization errors. In most cases (i.e. in smooth solution fields in analytic domains) it can be shown that the solution accuracy improves asymptotically with increase in the number of degrees of freedom.

\section{- Roundoff errors}

Since the computer handles variables usually through a finite number of words - a significant number of digits are rounded off to the next largest digit thus changing the values of the variables.

In adaptive FEA, the discretization errors are minimized using suitable error estimates.

\subsection{Error analysis and estimation}

In this section a typical elliptic PDE as used by Kelly et al (1983) and Gago et al (1983) is considered and the Galerkin method is used to discretize the weak form of the equations as it is done in traditional FEA. Subsequently, it is shown that a weighted residual expression of the strong form of the PDE can be reformulated on the discretized domain which 
gives rise to certain residuals that constitute the FE discretization errors. To arrive at the same results as Kelly et al (1983) and Gago et al (1983), a detailed derivation of the equations is presented below for the benefit of readers not conversant with functional analysis.

Let us consider a domain denoted by $\Omega$ bounded by $\Gamma_{D}$ and $\Gamma_{N}$ such that $\Gamma_{D} \cap \Gamma_{N}=0$ and $\Gamma_{D} \cup \Gamma_{N}=\Gamma$. Let the domain $\Omega$ be necessarily non-singular. Let a partial differential equation be defined in $\Omega$ as,

$$
-\nabla^{T}[a \nabla u]+b u+f=0,
$$

where, $u$ is an unknown function and $b$ and $f$ may be constants or functions depending upon the nature of the problem to be modelled. $\Omega \subset \mathfrak{R}^{3}$ (in the most general, i.e. 3-D case). The boundary conditions are given by the following equations,

$$
u=\bar{u}
$$

which are the geometric boundary conditions defined on $\Gamma_{D}$. For the current problem, homogeneous Dirichlet boundary conditions are used for which $\bar{u}=0$. The natural (Von Neumann) boundary conditions are given as:

$$
a \frac{\partial u}{\partial \mathbf{n}}=q
$$

which are defined on $\Gamma_{N}, \mathbf{n}$ being the unit normal vector drawn on the boundary away from the domain.

The weighted residual form of (1) may be written as,

$$
-\int_{\Omega} v \nabla^{T}[a \nabla u] \mathrm{d} \Omega+\int_{\Omega} b u v \mathrm{~d} \Omega+\int_{\Omega} v f \mathrm{~d} \Omega=0,
$$

where $v$ is a weighting function. On using the Gaussian divergence theorem on (1), the following equation is obtained,

$$
-\int_{\Omega} v \nabla^{T}[a \nabla u] \mathrm{d} \Omega=\int_{\Omega}[\nabla v]\left[a \nabla^{T} u\right] \mathrm{d} \Omega-\int_{\Gamma} v a \nabla u \cdot \mathbf{n} \mathrm{d} \Gamma .
$$

Assuming that the weighting functions $v$ actually have square integrable first derivatives and obey the homogeneous Dirichlet boundary conditions, (5), (2) and (3) yield,

$$
-\int_{\Omega} v \nabla^{T} u[a \nabla u] \mathrm{d} \Omega=\int_{\Omega}[\nabla v]\left[a \nabla^{T} u\right] \mathrm{d} \Omega-\int_{\Gamma_{N}} v q \mathrm{~d} \Gamma .
$$

From (6), the weak form of the problem is obtained as,

$$
\int_{\Omega u}\left[a \nabla^{T} u\right][\nabla v] \mathrm{d} \Omega+\int_{\Omega} f v \mathrm{~d} \Omega+\int_{\Omega} b u v \mathrm{~d} \Omega-\int_{\Gamma_{N}} v q \mathrm{~d} \Gamma=0 .
$$

In this context, the concept of a bilinear form is presented as,

$$
B(u, v)=\int_{\Omega}\left(L_{1} u M_{1} v+L_{2} u M_{2} v+\ldots\right) \mathrm{d} \Omega,
$$


where $u, v$ are functions in the same normed vector space and $L_{i}$ and $M_{i}$ are non-zero linear operators on $u$ and $v$. Using this relation in (7), we obtain,

$$
B(u, v)=\int_{\Omega}\left[a \nabla^{T} u\right][\nabla v] \mathrm{d} \Omega+\int_{\Omega} b u v \mathrm{~d} \Omega=-\int_{\Omega} f v \mathrm{~d} \Omega+\int_{\Gamma_{N}} v q \mathrm{~d} \Gamma .
$$

As stated previously, $v$ belongs to a space of functions which have continuous square integrable first derivatives and satisfy the homogeneous Dirichlet boundary conditions. Let this functional space be called $H_{D}$, and let a family of functions be $N_{i} \in H_{D}$. The functions $u$ are approximated as,

$$
\tilde{u}=\sum_{i=1}^{M} N_{i} \tilde{u}_{i}
$$

Thus, $B(u, v)=B\left(\tilde{u}, N_{i}\right)$

$$
B\left(\tilde{u}, N_{i}\right)=\int_{\Omega}\left[a \nabla^{T} \tilde{u}\right]\left[\nabla N_{i}\right] \mathrm{d} \Omega+\int_{\Omega} b N_{i} \tilde{u} \mathrm{~d} \Omega .
$$

Now, the functions $N_{i}$ are piecewise continuous functions over subdomains $\Omega_{i}$ where $\bigcup \Omega_{i}=\Omega$. Each of these subdomains $\Omega_{i}$ is bounded by the boundary $\Gamma_{i}$. The continuity of the functions $\tilde{u}$ are of the same order as of the weighting functions $N_{j}$ (BubnovGalerkin approach), which ensures that these two function families indeed belong to $H_{D}$. The only difference is that the previous function space was defined in $H_{D}(\Omega)$ while the current space is defined in $H_{D}\left(\Omega_{i}\right)$. Hence, the discretized equation may be represented as:

$$
B\left(\tilde{u}, N_{j}\right)=\sum_{i=1}^{n} \int_{\Omega_{i}}\left[a \nabla^{T} N_{j}\right][\nabla \tilde{u}] \mathrm{d} \Omega+\sum_{i=1}^{n} \int_{\Omega_{i}} b N_{j} \tilde{u} \mathrm{~d} \Omega .
$$

One important point to be noticed about this step is that discretization introduces some artificial boundaries in the system at which none of the boundary conditions are valid. Thus, at these zones some perturbations may arise if we revert back to the weighted residual form since the PDE is now redefined on a different domain (i.e. connected set of discrete subdomains). To demonstrate this, the first integral of (12) is integrated by parts by using the Gaussian divergence theorem as,

$$
\begin{aligned}
\int_{\Omega_{i}} \nabla^{T} N_{j}[a \nabla \tilde{u}] \mathrm{d} \Omega= & -\int_{\Omega_{i}} N_{j} \nabla^{T}[a \nabla \tilde{u}] \mathrm{d} \Omega+\int_{\Gamma_{P} \subset \Gamma}\left[a \frac{\partial \tilde{u}}{\partial \mathbf{n}}\right]_{\Gamma_{P}} N_{j} \mathrm{~d} \Omega \\
& +\int_{\Gamma_{K} \not \subset \Gamma} J\left(a \frac{\partial \tilde{u}}{\partial \mathbf{n}}\right)_{\Gamma_{K}} N_{j} \mathrm{~d} \Gamma,
\end{aligned}
$$

where, $J[a(\partial \tilde{u} / \partial \mathbf{n})]_{\Gamma_{K}}$ is the "jump" or discontinuity in fluxes across the element interface. This occurrence is solely due to the approximation made in the function $u$. Although only first order continuous functional approximation of $u$ needs to be done to satisfy the weak form of the equation, the first derivatives of these functions are not continuous across element boundaries which manifests itself in the PDE in the strong form. Thus, using (13) and (4), and rearranging the terms, the following equation is obtained as a complete weighted 
residual formulation of the problem using the approximate functions on the discretized topology of the domain $\Omega$.

$$
\begin{aligned}
\sum_{i=1}^{n}\left[\int_{\Omega_{i}} N_{j}\left[\nabla^{T}(a \nabla \tilde{u})+f+b \tilde{u}\right] \mathrm{d} \Omega\right]= & -\left[\int_{\Gamma_{P} \subset \Gamma}\left(a \frac{\partial \tilde{u}}{\partial \mathbf{n}}\right) N_{j} \mathrm{~d} \Gamma\right]_{\Omega_{i}} \\
& -\left[\int_{\Gamma_{K} \not \subset \Gamma} J\left(a \frac{\partial \tilde{u}}{\partial \mathbf{n}}\right)_{\Gamma_{K}} N_{j} \mathrm{~d} \Gamma\right]_{\Omega_{i}} \\
& +\left[\int_{\Gamma_{N}} N_{j} q \mathrm{~d} \Gamma\right]_{\Omega_{i}}
\end{aligned}
$$

From (14), which is also presented in Kelly et al (1983) and Gago et al (1983), it is evident that a new weighted residual (WR) form may be created when approximate functions for $u$ are used on the discretized domain $\Omega_{i}$, which creates some non-zero "residual" or error terms as will be shown in the next section. These are the discretization errors which are introduced in the FE formulation. From the new WR form in (14), let the substitute problem statement in the PDE form. (strong form) be constructed. There are practically three groups of integrals to be dealt with in this problem, i.e. those on the domain $\Omega$, those on the interface boundaries $\Gamma_{P}$ which are not a part of the boundary $\Gamma$ (and thus necessarily a part of $\Omega$ ) and the Von-Neumann boundaries $\Gamma_{N}$. In fact, (14) gives the terms arising out of the violation of the (domain) equilibrium and the natural boundary conditions and associates them to a spurious "jump" of traction values across the discretization interfaces arising due to the lower order approximation of the function $u$ by $\tilde{u}$. The domain integrand may be written as,

$$
\nabla^{T}(a \nabla \tilde{u})+b \tilde{u}+f=R
$$

where $R \neq 0$, since in general $u \neq \tilde{u}$. The terms on the Von Neumann boundary may be written as

$$
q-a \frac{\partial \tilde{u}}{\partial \mathbf{n}}=\bar{F}
$$

where $\bar{F}$ is a non-zero vector. The term on the interface boundary is given by:

$$
\left[J\left(a \frac{\partial \tilde{u}}{\partial \mathbf{n}}\right)_{\Gamma_{K}}\right]=\bar{B},
$$

where $\bar{B}$ is also a non-zero vector quantity. Thus, it can be remarked that $\bar{R}$ is the term arising out of the approximation of $u$ inside the domain, $\bar{F}$ indicates the violation of the natural boundary conditions due to the approximation of the function $u$ on the VonNeumann boundaries and $\bar{B}$ is the measure of discontinuity of the first derivatives of $\tilde{u}$ on the discretized boundaries arising due to the discretization of the domain and the functional approximation of $u$. From the above equations it is also clear that, in order to evaluate the error of discretization, all the three terms need to be considered for study. In structural mechanics applications the domain term indicates the violation of internal element equilibrium, the Von Neumann boundary terms indicate the errors in load modelling and the element interface integral gives the jump in stresses across the element boundary. 


\subsection{Substitute variational problem in error}

As seen in the previous section, discretization of the domain $\Omega$ and functional approximation of $u$ are responsible for perturbations of the basic PDE, the natural boundary conditions and the first-order derivative discontinuities of the function on discrete boundaries. The selection of the approximate function $\tilde{u}$ is guided by the requirements of the weak form of the functional which is different from the actual function $u$ whose continuity requirements are higher since it satisfies the strong form of the functional. However, the approximate function is always a subset of the original functional space. Following Kelly et al (1983), the bilinear form of the error from the equations derived earlier is established, as given below.

Let an error function $e$ be introduced such that

$$
e=u-\tilde{u} \text {. }
$$

If the function $e$ is introduced in the PDE (1) and the boundary conditions given by (2) and (3), then the following relations are obtained:

$$
-\nabla^{T}(a \nabla \tilde{u})+b \tilde{u}+f=R .
$$

Hence,

$$
-\nabla^{T}(a \nabla e)+b e+R=0 .
$$

The Dirichlet boundary conditions on $\Gamma_{D}$ become

$$
u=\bar{u} .
$$

The Von Neumann boundary condition is given on $\Gamma_{N}$ by the following equation,

$$
a \frac{\partial e}{\partial \mathbf{n}}=q-a \frac{\partial \tilde{u}}{\partial \mathbf{n}}
$$

Thus, the above set of equations pose a strong form of the substitute problem in $e$. Using the method of weighted residuals as done previously in an attempt to decompose the problem to its weak form (using the same basis functions $v \in H_{D}$ which obey the homogeneous geometric boundary conditions), the following equations are obtained:

$$
-\int_{\Omega} \nabla^{T}(a \nabla e) v \mathrm{~d} \Omega+\int_{\Omega} b e v \mathrm{~d} \Omega+\int_{\Omega} v R \mathrm{~d} \Omega=0
$$

Using the Gaussian divergence theorem on the first integral of the above equation we get,

$$
-\int_{\Omega} \nabla^{T}(a \nabla e) v \mathrm{~d} \Omega=\int_{\Omega} \nabla v\left(a \nabla^{T} e\right) \mathrm{d} \Omega-\int_{\Gamma} v\left(a \frac{\partial e}{\partial \mathbf{n}}\right) \mathrm{d} \Gamma .
$$

Since $v$ satisfies the homogeneous Dirichlet boundary conditions, the above equation decomposes to the following:

$$
-\int_{\Omega} \nabla^{T}(a \nabla e) v \mathrm{~d} \Omega=\int_{\Omega} \nabla v\left(a \nabla^{T} e\right) \mathrm{d} \Omega-\int_{\Gamma_{N}} v\left(a \frac{\partial e}{\partial \mathbf{n}}\right) \mathrm{d} \Gamma .
$$

Using the substitute Von Neumann equation given previously, the above equation may be modified as

$$
-\int_{\Omega} \nabla^{T}(a \nabla e) v \mathrm{~d} \Omega=\int_{\Omega} \nabla v\left(a \nabla^{T} e\right) \mathrm{d} \Omega-\int_{\Gamma_{N}} v\left(q-a \frac{\partial \tilde{u}}{\partial \mathbf{n}}\right) \mathrm{d} \Gamma
$$


Thus, replacing this condition into the weighted residual equation (23), the following equation is obtained,

$$
\int_{\Omega} \nabla v\left(a \nabla^{T} e\right) \mathrm{d} \Omega-\int_{\Gamma_{N}} v\left(q-a \frac{\partial \tilde{u}}{\partial \mathbf{n}}\right) \mathrm{d} \Gamma+\int_{\Omega} b e v \mathrm{~d} \Omega+\int_{\Omega} v R \mathrm{~d} \Omega=0 .
$$

From (9), the bilinear form $B(e, v)$ may be given as,

$$
B(e, v)=\int_{\Omega} \nabla v\left(a \nabla^{T} e\right) \mathrm{d} \Omega+\int_{\Omega} b e v \mathrm{~d} \Omega .
$$

In structural mechanics problems, the bilinear form $B(u, v)$ in $(9)$ represents some measure of the internal energy (strain energy) of the body, whereas the right hand side indicates the external work done by the applied loads. In virtual work type formulations, $v$ is the vector of virtual displacements, and the bilinear form indicates the internal virtual work done by the body as a response to the external virtual work done by the loads. In (28), therefore, $B(e, v)$ indicates some measure in the error in internal energy of the body and $v$ are general Galerkin weighting functions. The external perturbation given (on the nondiscretized domain) is due to $\bar{R}$ (equilibrium error on domain due to modeled $u$ ) and $\bar{F}$ (violated natural boundary conditions).

As shown in $\S 2.1$, let the domain $\Omega$ be discretized into subdomains (finite elements) $\Omega_{i}$ and thus introduce several discretized boundaries (interelement boundaries) $\Gamma_{K}$ distinct from the Dirichlet and the Von Neumann boundaries, i.e.

$$
\Omega=\bigcup \Omega_{i}+\bigcup \Gamma_{K}\left(\Gamma_{K} \not \subset \Gamma\right) \text {. }
$$

Using (29), and the discrete weighting function $v_{j}$ where $j$ indicates the index indicating the degree of freedom (discrete), (28) is recast as

$$
B\left(e, v_{j}\right)=\sum_{i=1}^{n}\left[\int_{\Omega_{i}} \nabla v_{j}\left(a \nabla^{T} e\right) \mathrm{d} \Omega_{i}+\int_{\Omega_{i}} b e v_{j} \mathrm{~d} \Omega_{i}\right] .
$$

Using the Gaussian divergence theorem to integrate the first domain integral in (30), the following relation is obtained,

$$
\begin{aligned}
\sum_{i=1}^{n}\left[\int_{\Omega_{i}} \nabla v_{j}\left(a \nabla^{T} e\right) \mathrm{d} \Omega_{i}\right]= & -\sum_{i=1}^{n} \int_{\Omega_{i}} \nabla^{T}(a \nabla e) v_{j} d \Omega_{i} \\
& +\sum_{\Gamma_{K} \not \subset \Gamma} \int_{\Gamma_{K}} J\left(a \frac{\partial \tilde{u}}{\partial \mathbf{n}}\right) v_{j} \mathrm{~d} \Gamma \\
& +\sum_{\Gamma_{P} \in \Gamma_{N}} \int_{\Gamma_{P}} v_{j} a \frac{\partial e}{\partial \mathbf{n}} \mathrm{d} \Gamma .
\end{aligned}
$$

Substituting (31) in (30), we obtain:

$$
\begin{aligned}
B(e, v)= & -\sum_{i=1}^{n} \int_{\Omega_{i}} \nabla^{T}(a \nabla e) v_{j} \mathrm{~d} \Omega_{i}+\sum_{i=1}^{n} \int_{\Omega_{i}} b e v_{j} \mathrm{~d} \Omega_{i} \\
& +\sum_{\Gamma_{K} \not \subset \Gamma} \int_{\Gamma_{K}} J\left(a \frac{\partial \tilde{u}}{\partial \mathbf{n}}\right) v_{j} \mathrm{~d} \Gamma+\sum_{\Gamma_{P} \in \Gamma_{N}} \int_{\Gamma_{P}} v_{j} a \frac{\partial e}{\partial \mathbf{n}} \mathrm{d} \Gamma
\end{aligned}
$$


From the above equations it can be seen that the term $\int_{\Gamma_{K}} J[a(\partial e / \partial \mathbf{n})] v_{j} \mathrm{~d} \Gamma$, actually decomposes to $\int_{\Gamma_{K}} J[a(\partial \tilde{u} / \partial \mathbf{n})] v_{j} \mathrm{~d} \Gamma$, since the exact solution $u$ does not have discontinuous first-order derivatives across the interelement boundary. Also, from the original partial differential equation of the system and the modelled equation it is obvious that the following relation holds,

$$
\begin{aligned}
& \int_{\Omega_{i}} \nabla^{T}(a \nabla e) v_{j} \mathrm{~d} \Omega_{i}+\int_{\Omega_{i}} b e v_{j} \mathrm{~d} \Omega_{i}=\int_{\Omega_{i}} \nabla^{T}(a \nabla(u-\tilde{u})) v_{j} \mathrm{~d} \Omega_{i} \\
& \quad+\int_{\Omega_{i}} b(u-\tilde{u}) v_{j} \mathrm{~d} \Omega_{i}
\end{aligned}
$$

since,

$$
\int_{\Omega_{i}} \nabla^{T}(a \nabla u) v_{j} \mathrm{~d} \Omega_{i}+\int_{\Omega_{i}} b u v_{j} \mathrm{~d} \Omega_{i}=-\int_{\Omega_{i}} f v_{j} \mathrm{~d} \Omega .
$$

Substituting (33) and (34) in (32),

$$
\begin{aligned}
B(e, v)= & \sum_{i=1}^{n} \int_{\Omega_{i}} \nabla^{T}(a \nabla \tilde{u}) v_{j} \mathrm{~d} \Omega_{i}-\sum_{i=1}^{n} \int_{\Omega_{i}} b \tilde{u} v_{j} \mathrm{~d} \Omega_{i}-\sum_{i=1}^{n} \int_{\Omega_{i}} f v_{j} \mathrm{~d} \Omega \\
& +\sum_{\Gamma_{K} \not \subset \Gamma} \int_{\Gamma_{K}} J\left(a \frac{\partial \tilde{u}}{\partial \mathbf{n}}\right) v_{j} \mathrm{~d} \Gamma+\sum_{\Gamma_{P} \in \Gamma_{N}} \int_{\Gamma_{P}} v_{j} a \frac{\partial e}{\partial \mathbf{n}} \mathrm{d} \Gamma .
\end{aligned}
$$

On grouping terms under the domain and boundary integrals and replacing the domain residual term by $R_{i}$, we get,

$$
\begin{aligned}
B(e, v)= & -\sum_{i=1}^{n} \int_{\Omega_{i}} R_{i} v_{j} \mathrm{~d} \Omega \\
& +\sum_{\Gamma_{K} \not \Gamma_{N}} \int_{\Gamma_{K}} J\left(a \frac{\partial \tilde{u}}{\partial \mathbf{n}}\right) v_{j} \mathrm{~d} \Gamma+\sum_{\Gamma_{P} \subset \Gamma_{N}} \int_{\Gamma_{P}}\left(a \frac{\partial e}{\partial \mathbf{n}}\right) v_{j} \mathrm{~d} \Gamma
\end{aligned}
$$

Due to orthogonality relations, the bilinear form $B(e, v)$ is zero. Thus, (36), which has also been derived by Kelly et al (1983) and Gago et al (1983), indicates that the error in displacements $e$ is such that a homogeneous form of (36) is satisfied for the whole domain. Since (36) becomes an identity, it cannot be solved directly and several assumptions are made to evaluate $e$. For example, as reported by Kelly (1984) and Kelly \& Isles (1989) it is assumed that the domain residual (indicated by $\bar{R}$ ) and the interelement traction jump actually self equilibrate over an element and the natural boundary condition violations are treated as traction jumps at $\Gamma_{N}$. In fact, (36) represents an enhanced FE equation, in which the domain term indicates the residual internal energy, the term on $\Gamma_{K}$ indicates the work done due to unbalanced internal forces and the term on the Von Neumann boundary $\Gamma_{N}$ indicates the work done due to residual forces on loaded edges. This implies that the global sum of the residual forces actually yields a measure of the equilibrium error due to discretization. Thus, the standard FE energy, if enhanced by this residual energy, may yield better results. This concept is used by Cantin et al (1978) and Cook (1982) to estimate a better stress distribution from a given set of FE results by iteratively improving displacements using the residual loads. 
(a)

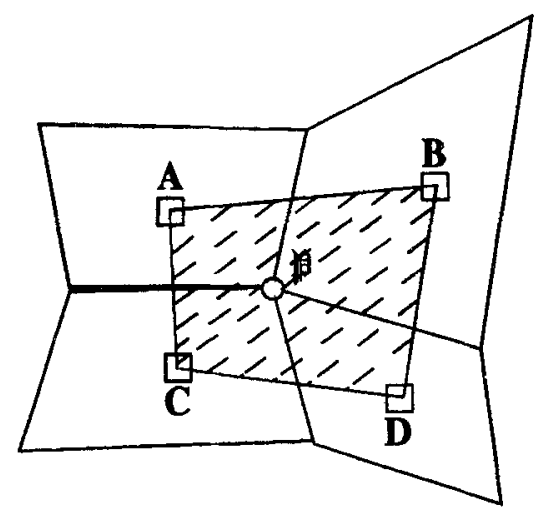

(b)

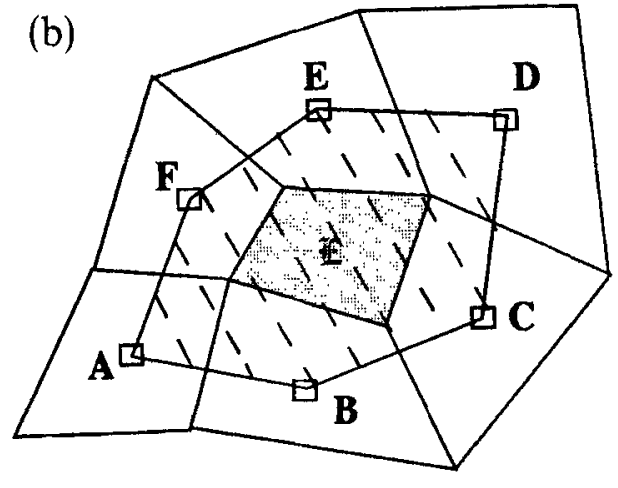

Figure 1. (a) - Node patch. (b) Element patch. A, B, C, D, E, F = centroidal superconvergent points of the elements, $\mathfrak{p}=$ patch assembly node, $\mathfrak{E}=$ patch assembly element.

\subsection{Error estimation procedures}

Several a posteriori type error estimates have been reported in literature. Among the most notable of these are the ones reported by Babuska \& Szabo (1982), where the residual form of the estimate is considered. Zienkiewicz \& Zhu (1987) designed a best guessed stress type error estimate based on least square smoothing of the stresses. More recently, Zienkiewicz \& Zhu (1992) reported a superconvergent error estimate based on patchwise stress recovery. The method was enhanced by Wiberg and coworkers (Wiberg \& Abdulwahab 1993; Wiberg \& Li 1994; Wiberg et al 1994) and Blacker \& Belytschko (1994) who used equilibrium and natural boundary condition residuals together with conjoint polynomials to derive an asymptotically exact estimate.

The development of such a posteriori error estimates is focussed on two aspects. First, a smoothed stress distribution needs to be extracted from the FE stresses and next, a proper refinement criteria needs to be designed which determines the new element size for the given error percentage. The errors are usually computed as a measure of the difference between the FE stresses and the smoothed stresses.

In the patchwise stress recovery method, a patch of elements is selected around the node as shown in figure 1a. The unknown smoothed stress variation over this patch is assumed to be

$$
\sigma^{*}=[1, x, y, x y]\{a\}
$$


where $\{a\}$ is a vector of undetermined coefficients. The discrete $L_{2}$ norm of the stress difference is considered to be the stress error functional, $\Pi_{\sigma}$ :

$$
\Pi_{\sigma}=\sum_{i=0}^{N P}\left[\sigma^{*}-\sigma_{h i}\right]^{T}\left[\sigma^{*}-\sigma_{h i}\right],
$$

where, $N P=$ number of superconvergent sampling points on patch, $\sigma_{h i}=$ superconvergent FE stress on $i$ th sampling point.

Equation (38) is valid only in the shaded area in the patch as shown in figure 1a. It may be noted that this equation is a least square estimation of the smoothed stresses from the superconvergent $\mathrm{FE}$ stresses $\sigma_{h i}$. On differentiating this functional with respect to the undetermined coefficients, we get the following set of linear equations,

$$
\sum_{i=0}^{N P}\left[\left[P_{i}\right]^{T}\left[P_{i}\right]\right]\{a\}=\sum_{i=0}^{N P}\left(\left[P_{i}\right]^{T} \sigma_{h i}\right),
$$

where,

$$
\left[P_{i}\right]=\left[1, x_{i}, y_{i}, x_{i} y_{i}\right]
$$

Now, let us consider (36) again. Let the error in displacement $e$ be replaced by

$$
e=u-u^{*}
$$

where $u$ is the exact displacement and $u^{*}$ is the displacement corresponding to the smoothed solution. Thus, the right hand side of (36) implies that the smoothed solution does not produce any equilibrium residual on the domain or any interelement residual on FE edges or any boundary residual on the Von Neumann boundaries. These conditions are thus used as constraint equations to enhance the functional $\Pi_{\sigma},(38)$, as

$$
\begin{aligned}
\Pi_{\sigma}= & \sum_{i=0}^{N P}\left[\sigma^{*}-\sigma_{h i}\right]^{T}\left[\sigma^{*}-\sigma_{h i}\right]+\beta_{1} \int_{\Omega_{p}}\left[\nabla^{T} \sigma^{*}-f\right]^{T}\left[\nabla^{T} \sigma^{*}-f\right] \mathrm{d} \Omega_{p} \\
& +\beta_{2} \int_{\Gamma_{p}}\left[[N]\left(\sigma^{*}\right)-\tilde{t}\right]^{T}\left[[N]\left(\sigma^{*}\right)-\tilde{t}\right] \mathrm{d} \Gamma_{p}
\end{aligned}
$$

where, $\beta_{1}, \beta_{2}=$ penalty coefficients, $N P=$ number of superconvergent sampling points on patch, $\left[\nabla^{T} \sigma^{*}-f\right]=$ equilibrium residual, $\Omega_{p}=$ patch domain, $[N]\left(\sigma^{*}\right)-\tilde{t}=$ Von Neumann residual, and $\Gamma_{p}=$ Von Neumann boundary on patch.

The term on the interelement boundary is omitted because the smoothed stress polynomial is continuous over the patch. Equation (41) was also presented by Wiberg et al (1994) and Blacker \& Belytschko (1994) but no strict justification was given regarding the enhancement of the basic stress functional by the equilibrium and the natural boundary condition enhancements. Thus, (36) represents the basic relation which is used to extract smoothed stresses in most of the published a posteriori error estimators either in direct form or as a constraint condition to enhance a least square stress functional given in (38).

The use of the augmented patch-based stress extraction methods has some mathematical inconsistencies in setting up of the limits of integration of the equilibrium and the Von Neumann residuals. It is seen that only a part of the patch is influenced by the 
least square polynomial (indicated by hatchmarks in figure 1a) which implies that the sampled values of $\sigma^{*}$ in zones exterior to this part cease to be reliable. This implies that the integration limits of the equilibrium and the Von Neumann residuals cannot be over the entire patch. Recently, Wiberg et al $(1994,1995)$ proposed the element patch (figure 1b) where such problems do not occur as the integration limits cover the entire element. However, as it is difficult to compute a least square projection of the stresses over the element patch, Wiberg et al $(1994,1995)$ use a least square displacement projection technique from which the stresses are computed by using the strain displacement and constitutive laws. However, as the QUAD4 element does not possess superconvergent displacement points, it is not practicable to extract superconvergent stresses from an enhanced displacement field directly although the enhanced displacement field is constrained by (36).

In this context, Mukherjee \& Krishnamoorthy (1996) have presented the element patchbased superconvergent error estimate which uses a least square fit of an enhanced stress polynomial and the penalty constraints of (36) are applied directly. Unlike Wiberg et al $(1994,1995)$, no displacement projection is done and thus the superconvergent nature of the stresses are guaranteed. The examples in this paper are solved by using this estimate.

\section{Automatic mesh generation procedures}

A major part of the effort in the adaptive FE process lies in the mesh generation procedure. Good reviews of mesh generation schemes may be found in Buell \& Bush (1973), Thacker (1980), and Ho-Le (1988). The available mesh generators now in use may be generally classified into two groups, i.e. mapped and automatic mesh generators. In the mapped mesh generation process the problem domain is usually manually decomposed into a set of mappable regions which are mutually non-intersecting. A mapping technique, usually an isoparametric or a transfinite procedure, is employed to explicitly or implicitly handle a set of geometric representations within each mapped region. These representations are defined in terms of the information specified on the boundaries of the subregion. More specifically, the isoparametric scheme is used to interpolate points in the subregion domain, while collocating at discrete points on the subregion boundary, and the transfinite mapping method interpolates points in the subregion domain, while collocating globally on the subregion boundaries. Thus, creation of transitions is impossible unless special measures are employed and, although these methods are fast, they are not flexible enough for local control.

Automatic or unstructured mesh generators are generally boundary based, i.e. the boundary definition of the meshable object is taken as the starting point of the mesh generator, and as the generation procedure progresses, the meshable domain geometry also changes continually. Thus, at every step of element generation, the geometry of the unmeshed domain needs to be evaluated. Hence, even though these processes have better mesh control and are more flexible, they are computationally more intensive. Also, the storage requirements for the unstructured mesh generators are larger as both connectivity and coordinates of the nodes need to be stored, whereas in case of mapped mesh generators only the coordinates need to be stored. 
Thus, it was commented in Krishnamoorthy et al (1995) that the motivation for the development of a new mesh generation is to design a system which incorporates the computational efficiency of the mapping techniques and the flexibility and control characteristics of unstructured mesh generators. Keeping this in mind, a new method of quadrilateral mesh generation was proposed (Krishnamoorthy et al 1995), called Meshing by Successive Superelement Decomposition (MSD), which was shown to be composed of two parts the Approximate Skeletal Method which automatically decomposes the problem domain into a set of mappable, topologically simple superelements and the Meshing by Successive Decomposition, which is a recursive quadrilateral mesh generation scheme acting on the individual superelements.

\subsection{Approximate skeletal method}

The theoretical basis of the approximate skeletal method is the generation of medial axes of objects, which is used for object recognition in pattern recognition theory. The implementation details of this technique are presented by Krishnamoorthy et al (1995). The theoretical basis of the method is briefly discussed in the following sections.

3.1a Medial axis transforms: In pattern recognition theory, a skeleton or a medial axis or a symmetric axis of an object is defined as the locus of those points which are minimally equidistant from any two boundary points of the object - in general the method for generating the skeleton is usually referred to as the Medial Axis Transform (MAT) technique or the Symmetric Axis Transform (SAT) technique. The existence of skeletons for various biological shapes and their use for shape description was first proposed by Blum (1967). Shapes are normally described by their boundaries - however, in MAT, the shape description of objects include the interior (and exterior) of the object by defining a primitive called a maximal disk. Hence the description of an object consists of two primitives viz. the medial axis (MA) and the maximal disk (MD). The locus of the centre of the MD is the MA itself and the radii of the MD form an envelope which describe the boundary of the object.

The flexibility and the generality of shape recognition by MAT cannot be overstressed. For example, the shape features which are shown in figure 2 are identified by simple local perturbations in the MA or the MD. In the case of a pinch, the "noise" in the MA proves its presence. The worm, wedge, cup and flare are all characterized by local curvature values of the radius function of the MD. In specific cases, like the worm, the curvature change is zero, in all other cases it is non-trivial. Thus, by using MA and MD, not only the boundary features but also the width properties of the object may be identified.

Mathematically, in MAT, an intrinsic coordinate system is used to define any twodimensional object. Given a closed boundary $\Delta$ of a domain $\Omega$, the Euclidean distance $d(x, \Lambda)$ from any point $x$ to a set of boundary points $\Lambda$ is given by

$$
\mathrm{d}(x, \Lambda)=\min [\mathrm{d}(x, y): y \in \Lambda] \text {. }
$$

It is clear that for some points, more than one boundary point satisfies this minimal distance criteria and the locus of such points is the MA of the system. Let this MA be designated 

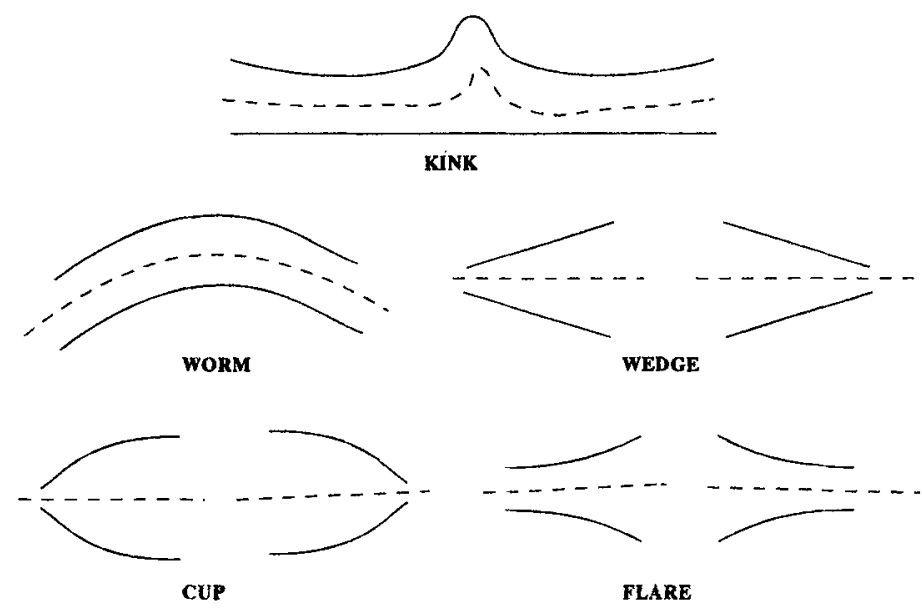

Figure 2. Some elementary shape descriptors based on width properties and their MA.

$S$; then a function $f(x)$ can be defined such that it maps $\Lambda$ into a set of non-negative real numbers $\rho \in \mathbf{R}$, where $\mathbf{R}$ is the space of all non-negative real numbers as

$$
f(x)=\mathrm{d}(x, \Lambda)
$$

From (42) and (43) as given above, it is clear that $f(x)$ is the radius function or the disk function of the domain. The value of this function for any $x$ on $S$ gives the measure of the radius of the MD at $x$.

It can be shown that the MA of a shape and the Voronoi diagram of its edges are interrelated. In fact, they are identical for convex domains - however for non-convex domains, the Voronoi diagram is different.

Geometrically, the MA is composed of several connected segments joined at a set of points called the skeleton nodes which are also called branch points (Blum 1967). Hence, the MA of a 2D object is a one-dimensional planar graph without any area. The MAT of objects can exist even outside the domain as shown in figure 2. If the domain itself is considered as a hole in a very large bounded circle, then many properties of the internal MAT are also seen as that of the external MAT. However, external MATs have two distinct properties of their own, viz. for convex shapes external MATs do not usually exist and, even if they do, they are not connected.

Interior MA can be used in pattern recognition as shown by Blum \& Nagel (1973) and automatic mesh generation, as reported by Gursoy \& Patrikalakis (1992) and Tam \& Armstrong (1991), while exterior MA is used for motion planning and mesh generation for CFD applications. Figure 3 shows the interior and the exterior skeletons of an arbitrary domain.

The analysis of MATs of continuous shapes was investigated by Blum (1973), Calabi \& Hartnett (1968a, 1968b), and Nagel \& Blum (1976). Computer implementation and discrete MA theory was developed by Montanari (1968). Besides these, the works of Lee (1982) and Bookstein (1979) are also noteworthy. 


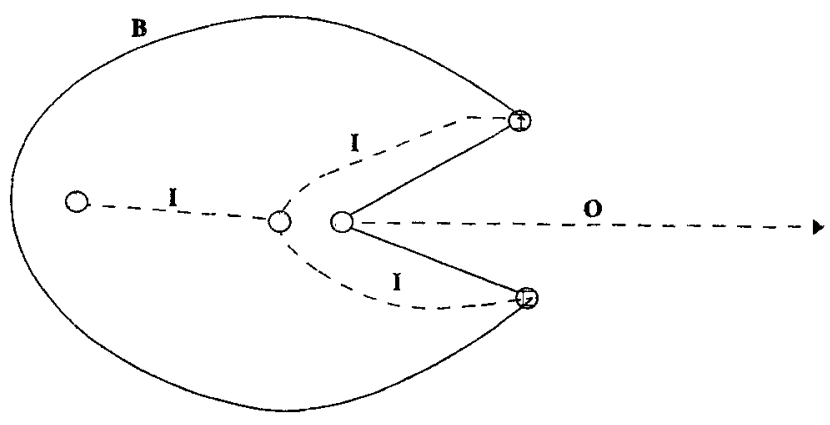

Figure 3. Outer and inner MAT of a domain. $\mathrm{O}=$ outer medial axis, $\mathrm{I}=$ inner medial axis. $\mathrm{B}=$ boundary.

3.1b Domain decomposition using MAT: While addressing the issue of domain decomposition, the methods used for generating MA branches should be highlighted. In the present case, Krishnamoorthy et al (1995) presented the equations of the MA in simple parametric form since the domain boundaries could be represented by analytic equations. In pattern recognition theory, thinning algorithms and in CAD systems geometric search techniques are usually used for generating the MA branches (Turkiyyah \& Fenves 1988). In case of $\mathrm{FE}$ mesh generation applications, domain feature extraction is not carried from the MA and hence a mathematically accurate MA extraction is not necessary. This is the basis for the method presented in Krishnamoorthy et al (1995), where simplified representations of boundary and the MA branches ensure a large computational saving in the domain decomposition process.

The assumptions introduced in Krishnamoorthy et al (1995) produce no major perturbation of the MA. All curved boundary segments are represented as a union of line segments. This simplifies computations since the MAT of straight edges are only first- or seconddegree curves. In fact, if boundary representations are handled by quadratic polynomials, the MA becomes a quartic polynomial. However, in the proposed algorithm, the MA is handled by piecewise continuous quadratic polynomials, consistent with the simplified boundary representations.

The MA branches (also referred to as skeletal curves or radial lines), which are rays traced from skeleton nodes to nearest boundary segments, and the boundary segments themselves decompose the domain into a set of non-intersecting, topologically simple superelements which are considered individually for mesh generation. The radial lines indicate the radii of the MD centred at the skeleton nodes.

The superelement generation process is thus composed of four steps as shown below.

- Generation of equidistant curve.

- Generation of skeletal curve segments. 


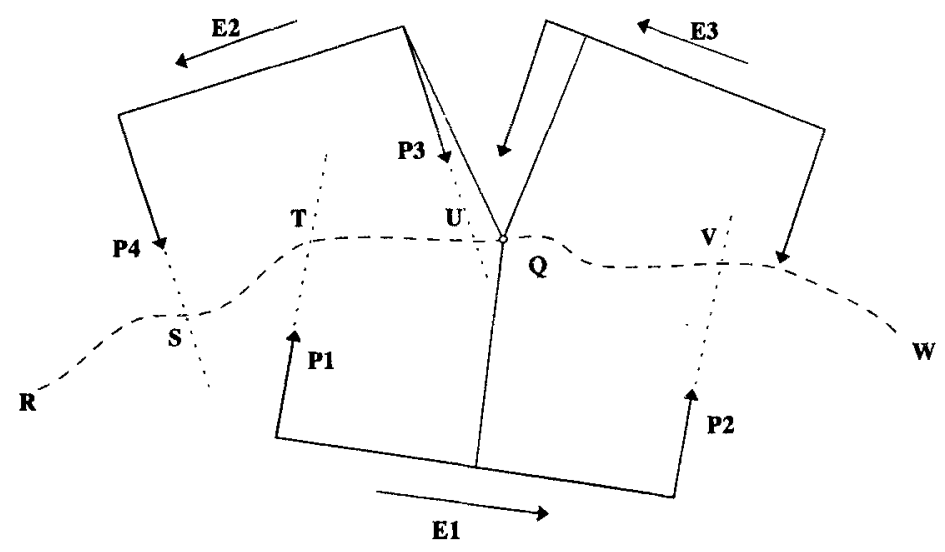

Figure 4. Equidistant and skeletal curves.

- Ray tracing from skeleton node to generate radial lines.

- Geometric merging processes to correct distorted superelements.

3.1b(i) Generation of equidistant curve - An equidistant curve for a pair of line segments is defined as the locus of all the points which are equally distant from these segments. In figure 4, the segments E1 and E2 are used to generate the equidistant curve RW which consists of five piecewise continuous curve segments RS, ST, TU, UV and VW. The discontinuities of the curve are marked by the perpendiculars P1, P2, P3 and P4. The equidistant curve is a piecewise continuous quadratic polynomial in these five segments.

The coordinate systems and the computation procedures for the points on the equidistant curve may be found in Krishnamoorthy et al (1995).

3.1b(ii) Generation of skeletal curves - In the present work, the boundary primitives are straight lines, a chain of straight line segments representing a curved edge and reentrant vertices. The skeletal curve is a subset of the equidistant curve which is constrained by the interference of a third boundary primitive in accordance with (43). Thus, the skeletal curve segment is basically a branch of the MA and the union of these segments gives the MA of the whole domain. In figure $4, Q$ is a typical skeleton node generated by edge segment $\mathrm{E} 3$ and the segment SQ is a MA branch (skeletal curve segment) of the doublet defined by (E1, E2).

Thus, the MA is composed of several such individual segments which are bounded by these skeleton nodes and, since each is independent of the effect of another, they are unique, disjoint and complete. Since a unique pair of boundary segments is used to generate each segment, such doublets are also unique, disjoint and complete.

3.1b(iii) Subregion decomposition - The decomposition of an object into meshable subregions is preceded by the generation of the shape primitives, which are derived from the medial axis and are discussed in the next section. 
3.1b(iv) Shape primitives - In this context, the concept of a shape primitive is introduced next, as proposed in Krishnamoorthy et al (1995). Let $\Omega$ be a bounded planar domain with a boundary $\Lambda$, then following earlier definitions, $\mathrm{d}(x, y)$ is the Euclidian distance function from a point $x \in S$ to a point $x \in \Lambda$ where $S$ is the MA. Thus $\mathrm{d}(x, y)$ is also the radius function of the MD at $x$. So, if a normalized coordinate system $t$ is used for each MA branch and boundary segment, then $D(t)$ becomes the normalized Euclidian distance function and indicates the lengths of the perpendiculars from the boundary segments onto the MA branch. At the skeleton node, there are three such equal perpendiculars from the two generating and one constraining boundary edge.

As the MA is defined as the locus of the centres of such maximal disks which are tangential to the boundary segments, the distance function may be defined as the locus of the point of tangency of the MD to the boundary. In this context, let us introduce one more constraint in the definition of the MA; allowing the MD to move inside $\Omega$ such that the bounding box to the MD has at least two opposite edges lying on the boundary $\Lambda$. It may be observed that the locus of the centre of MD, $\bar{S}$, is only a subset of the MA, $S$. This new shape attribute of the domain $\Omega$ is now called the shape primitive (SP) of the domain. In geometrical terms, it is clear that the MA is a list of branches bounded by skeleton nodes and boundary nodes, whereas the SP is a list of branches bounded by skeleton nodes only. It may be noted that the algorithm for the generation of the SP differs markedly from the grassfire algorithm as proposed by Patrikalakis \& Gursoy (1990).

Previously, it was stated that the MA is a subset of the edge Voronoi diagram, and now the SP is presented as a subset of the MA.

Recently, Reddy \& Turkiyyah (1995) presented the trimmed skeleton which is identical to the SP as proposed earlier by the authors.

3.1c Skeleton node classification and ray tracing methods: The branches of the shape primitives are identified by the type of skeleton nodes they are bounded by. The skeleton nodes are, in turn, identified by the number and configuration of the radial lines that can be traced from them to the object boundary $\Lambda$. In general, the rays from the skeleton node to the object boundary segments depend upon the relative positions of these segments in the 2D plane. For example, if three boundary edges are adjacent to each other, then the SP branch that is generated is itself a skeleton node. This is the typical branch node in MA where the MD touches $\Lambda$ at three places, thus in the present case, three radial lines can be drawn from such nodes, hence such nodes are named triple ray type nodes. Similarly, in cases where at least two edge segments are adjacent or all three edges are non adjacent, triple ray type nodes are generated. In cases where the two edges are nonadjacent and are the generators of an SP arc, usually double ray type nodes are introduced which are basically the normal nodes in MA where the MD touches $\Lambda$ at two points. It is to be noted that the double ray type node contradicts the definition of the skeleton node, but in case of domain decomposition applications, where the quality of the generated superelements is important, such double ray type nodes need to be introduced in places where the generating edge segments change curvature or, in general, possess large curvature. In the presence of reentrant corners pseudo double ray type nodes are introduced which remove the concavity. The generation of these nodes creates the geometric semiligatures as stated in Blum \& 
Nagel (1973). In the presence of end zone arcs, pseudo double ray type or pseudo triple ray type nodes are generated depending on the angle which the arc subtends in the domain. Thus, as a deviation from the usual MAT, the end points are replaced by either normal or branch points.

Thus, each branch of the SP is bounded by exactly two of these node types as mentioned above. Each node type in turn is represented by the radial lines traced from them to the boundary of the object. Hence, in such a representation it is possible to maintain all the shape properties of the parent domain. In the traditional MA methods, the width properties of the object are represented by the MD while the axial properties of the object are represented by the branches of the MA, which is an intrinsic coordinate system independent of the external Cartesian system used to represent the object. In the proposed approach, keeping in mind the subsequent domain decomposition, the representation is done by the branches of the SP and the skeleton nodes which specify the radial lines - so the intrinsic coordinate system in this case is different from the one as given in Nagel \& Blum (1976).

3.1d Decomposition into superelements: The SP branches of a domain, together with the radial lines and the boundary edge segments form the basis of the subdivision of the domain into superelements. In fact, superelements may be grouped into two classes, viz. end-zone superelements and body superelements. The end-zone superelements are quadrilaterals typically bounded by two adjacent boundary segments and two radial lines, and with four nodes of which one node is a skeleton node while the other three are boundary nodes. These are generated in the regions where three boundary edges are adjacent, and the skeleton node in these superelements usually marks one extremity of the SP of the domain. The body superelements may be triangular or quadrilateral and are characterized by one SP branch, two radial lines, two skeletal nodes and at least one boundary node. These may be formed anywhere inside the domain along the SP of the body. Thus the set of these two types of superelements completely decompose the object into a set of non-overlapping, topologically simple subregions.

As stated in the earlier section, the proposed intrinsic coordinate system of the object represents the object as a set of piecewise continuous SP segments bounded by the skeleton nodes. For representing the width, radial lines are associated with skeleton nodes. The superelement generation is shown as a natural extension to this object recognition strategy and the superelements include the boundary definition of the object together with the information on the interior of the object in terms of the skeleton nodes, SP branches and radial lines. Thus, the superelements encapsulate all the geometric features of the domain and may be used to represent the domain in all further computations.

The decomposed representation of the domain as presented above is important with respect to the mesh generation and attribute handling point of view. The decomposition essentially implies that if any engineering feature is assigned to any of the superelements, then this feature would also be the property of any geometric entity that is extracted from this superelement. In a more global term, if the same feature is assigned to any part of the parent domain, then all superelements which compose that specific part will also inherit that feature. Subsequently, any geometric entity which is extracted from these superelements will in turn inherit this particular feature too. As an example, if a certain boundary condition 
is applied on a part of $\Lambda$, then all superelement edges which contain that part of the boundary inherit the same boundary condition. In turn, all element nodes which may be generated on all such superelement edges also inherit that boundary condition, irrespective of the nature of the FE mesh. Thus, a hierarchic relation is created in the proposed model, which is suitable for adaptive analysis applications, since at every stage of analysis the mesh is modified requiring redistribution of attribute data.

3.1e Control and correction of superelements - the merging process: The quality of the superelements usually affect the quality of the elements generated inside them. If the set of boundary segments of $\Omega$ include reentrant vertices or short boundary segments, then distorted superelements are generated which, in turn, may be responsible for large element distortions within them. It has also been noticed by the authors that for convex vertices with large included angles, large taper distortions usually occur. The merging process rectifies this anomaly by moving skeleton nodes toward one another to modify such geometry.

In Krishnamoorthy et al (1995), two such merging procedures viz. parallel shift and angular shift corrections were proposed based on the movements of skeleton nodes to correct the distorted superelements. A set of rules for moving the node was also laid out.

\subsection{Meshing by successive decomposition}

As stated earlier, in adaptive mesh generation procedures, local mesh control is of prime importance, hence structured mesh generators which have control only on the superelement edges are not very useful.

Among the more well-known quadrilateral mesh generators, the schemes by Zhu et al (1991), Talbert \& Parkinson (1990) and Blacker \& Stevenson (1991) are notable. The technique presented here overcomes the shortcomings of conventional mapping techniques and does not involve the computational complexities of other unstructured quadrilateral mesh generators either.

In the present method, the superelements are divided recursively using discrete curve segments generated by transfinite interpolation. Nodes are generated recursively on these segments from a proposed background-grid to ensure complete internal local control of the mesh density. Multiple splitting methods are introduced to create transitions leading to mesh gradation within the subdomain.

The general procedure for the proposed mesh generator is based on recursive splitting (with transitions). The procedure starts by discretization of the superelement boundaries into an even number of segments (Heighway 1983). Then the splitting procedure starts from the boundary to divide the superelement into a set of 2,3 or 4 children superelements and accordingly the edges are also split. The new edges thus generated are now discretized by nodes and the children superelements are considered for an even number of segments. The procedure continues until the last child superelement edge may not be segmented any further. Then this superelement is recognized as a quad 4 element and the procedure continues till all the children superelements have been thus transformed. The 
node-spacing information is obtained from a proposed background grid which is created from a postprocessed FE solution, i.e. it contains the nodal spacing data corresponding to some error-tolerance norm. A garbage collection algorithm was developed to handle the recursive operations. A detailed description of this process may be found in Krishnamoorthy et al (1995).

\section{Refinement criteria - a new $h$-refinement strategy}

In this section the authors propose a new $h$-refinement strategy which deviates from the conventional refinement strategy and it is shown that the new strategy yields a better convergence rate for the problems solved.

\subsection{Conventional h-refinement criteria}

The conventional $h$-refinement procedure was first proposed by Zienkiewicz \& Zhu (1987). Error estimators of the elements were used to construct new element sizes at the centroid of the old elements on the basis of the assumption that the most optimal mesh is the one where the error is equally distributed among all the elements of the mesh. The general derivation for this is as follows.

Let a set of elliptic PDEs defined over a typical domain $\Omega$ be

$$
\mathbf{L u}=\mathbf{q},
$$

subject to the boundary conditions:

$$
\begin{aligned}
u & =\bar{u} \text { on } \Gamma_{D} . \\
\partial u / \partial \mathbf{n} & =\bar{q} \Gamma_{N} .
\end{aligned}
$$

In typical linear elasticity applications, $\mathbf{L}$ is a linear differential operator, $\mathbf{u}$ is the unknown function of displacements, $\mathbf{q}$ is the body force term, and (39) is the equilibrium equation. If the maximum element diameter of a FE discretization is $h_{e}$, and the degree of the interpolating polynomial is $n$, then, the error in displacement is given by:

$$
E_{u}(h)=O\left(h^{n+1}\right) \leq C h^{n+1},
$$

where $C$ is some constant.

If the stresses and the strains (i.e. general derivatives) are given by the $m$ th derivative, then, we get

$$
E_{\sigma}(h)=O\left(h^{n-m+1}\right) .
$$

Then the error bounds of the strain energy, which is a quadratic functional of the displacement, become

$$
E_{e}(h)=O\left(h^{2(n-m)+1}\right) .
$$

In another form, the norm of the error in energy becomes

$$
\left\|E_{e}\right\|=O\left(h^{(n-m)+1}\right),
$$


where the global energy norm error, $\left\|E_{e}\right\|$ is computed as

$$
\left\|E_{e}\right\|=\left[\int_{\Omega}\left[\sigma^{*}-\sigma_{h}\right]^{T}[D]^{-1}\left[\sigma^{*}-\sigma_{h}\right] \mathrm{d} \Omega\right]^{0.5} .
$$

The local energy norm error is given by

$$
\left\|E_{e}\right\|_{i}=\left[\int_{\Omega_{i}}\left[\sigma^{*}-\sigma_{h}\right]^{T}[D]^{-1}\left[\sigma^{*}-\sigma_{h}\right] \mathrm{d} \Omega\right]^{0.5} .
$$

The local and global energy norm errors are related as:

$$
\left\|E_{e}\right\|^{2}=\sum_{i=1}^{M}\left\|E_{e}\right\|_{i}^{2}
$$

where, $\sigma^{*}=$ smoothed stress, $\sigma_{h}=$ FE stress, $\Omega_{i}=$ element domain, $M=$ total number of elements.

The convergence order of the global energy norm error is the same as that of the errors in the stresses, hence an accurate stress projection method will automatically accelerate the convergence in the energy norm (or $L_{2}$ norm). Thus,

$$
\left\|E_{e}\right\| \leq C_{1} \mathrm{O}\left(h^{(n-m)+1}\right),
$$

where $C_{1}$ is a constant depending on element aspect ratio, quadrature rule etc. In fact this constant may be shown to be dependent on some norm of the displacement function $u$. As the element size tends toward zero $(h \rightarrow 0)$, the above equation tends to an equality with the bound given in an asymptotic manner. It has been shown in Babuska \& Szabo (1982) and Zienkiewicz \& Zhu (1992) that if stress extraction is based on superconvergent principles, then indeed (52) is true. If $N$ is the number of global degrees of freedom in the system, and $K$ is an arbitrary constant, then, we get

$$
N=K / h^{2} \text {. }
$$

Replacing in (44), the following condition is obtained :

$$
\left\|E_{e}\right\| \leq C_{1} N^{[1+(n-m)] / 2}
$$

or

$$
\left\|E_{e}\right\| \leq C_{1} N^{-p / 2}
$$

where $p=1-n+m$.

The bound given above is valid for smooth solutions in regular domains. However, in case of non-regular domains, the following modification is suggested,

$$
\left\|E_{e}\right\| \leq C_{1} N^{-\min [\lambda, p] / 2},
$$

where $\lambda$ is a parameter whose value depends upon the singularity in the domain. Usually, for elastic problems, $\lambda$ is 0.50 for closed cracks and 0.71 for a $90^{\circ}$ corner.

Now, let $\bar{\eta}$ be the target global error fraction and $\eta$ be the actual relative percentage error, then 


$$
\eta=\frac{\left\|E_{e}\right\|}{\left(\left\|E_{e}\right\|^{2}+\|\tilde{u}\|^{2}\right)^{0.5}}
$$

where, $\|\tilde{u}\|$ is the strain energy of the body. Obviously, this equation is valid in the local as well as the global form. Hence, both local and global relative percentage error energy measures can be computed.

Let a reference norm be given by the following

$$
\|R\|=\|u\| / M^{0.5},
$$

where $\|u\|$ is the energy computed on the basis of an improved stress field and $M$ is the number of elements of the system. Thus, the upper bound of the (elementwise) local error estimate is set as

$$
\left\|E_{e}\right\|_{i} \leq \vec{\eta}\|R\|
$$

It has been shown by Zienkiewicz \& Zhu (1987) that if the reference norm is based on the local energy level then usually overrefinement may occur. So, a global energy based reference norm is normally used. The above equation implies that the element level energy error reaches a fraction of the reference energy equally over all elements as $h$ tends toward zero. Thus, in the limit, the optimality condition is reached when all the elements have equal amounts of error in energy.

The size indicator is defined as the following:

$$
\xi_{i}=\left\|E_{e}\right\|_{i} / \bar{\eta}\|R\|
$$

where, $\xi_{i}$ is the size indicator used to change the size of the element as

$$
h_{i}^{\text {new }}=h_{i}^{\text {old }} / \xi_{i}^{\lambda},
$$

where $h_{i}^{\text {new }}$ and $h_{i}^{\text {old }}$ are the new and old element sizes respectively.

\subsection{Proposed h-refinement criteria}

In this proposed $h$-refinement criteria, both the reference norm and the target global error fraction are modified. The reference norm in this case is the weighted average of the energy norm which is computed as,

$$
\|W(u)\|=\sum_{i}^{M}\left(\|u\|_{i}{A_{i}}^{0.5}\right),
$$

where $\|u\|_{i}$ is the local element level strain energy computed by an improved stress field and $A_{i}$ is the area of element $i$.

The global energy density may be defined as

$$
D_{g}=\|u\| / A^{0.5},
$$

where, $A$ is the area of the domain.

The local energy density is defined as

$$
D_{i}=\|u\|_{i} / A_{i}^{0.5} \text {. }
$$


Thus, the modification to the target global error fraction is given as

$$
\eta_{i}=\bar{\eta}\left[D_{g} / D_{i}\right]^{\alpha},
$$

where $\alpha$ is a parameter which lies between 1.00 and 1.25 for most problems. The equation given above decomposes to the original expression for $\bar{\eta},(57)$, when $\alpha$ is zero. In physical terms the above equation reduces the modified target global error fraction where the energy density of an element exceeds the energy density of the system. Thus, automatically, this modification forces the element sizes to be smaller where there are large stress excursions.

The modification to the reference norm is given as

$$
\|R\|_{\bmod }=\|W(u)\| / A^{0.5},
$$

where, $\|\cdot\|_{\text {mod }}$ is the modified reference norm. It may be noticed that instead of the average of the energy which was computed in (58), this norm computes the weighted average of the energy. In the case where all the elements are of equal size, the modified reference norm decomposes to the original reference norm. If $L_{2}$ norm is used instead of the energy norm and Von Mises stresses are used instead of Cauchy's stresses, then this refinement criteria changes into the adaptive accuracy scheme as reported by Grosse et al (1992). The weighted average energy norm was first discussed in the context of structural shape optimization by Bugeda \& Oliver (1991),

$$
\left\|E_{e}\right\|_{i} \leq C_{1} \beta_{i} \frac{-1}{\lambda}\|R\|,
$$

where $\left\|E_{e}\right\|_{i}$ is usually the local error in energy, the constant $C_{1}$ is the user defined target global error fraction, $\beta_{i}$ is some measure of the element size, $\lambda$ is a function of the convergence rate and $\|R\|$ is the reference norm.

In this context it may be relevant to discuss some other previously published refinement criteria, which are either SED-based or SED-enhanced, and their differences with the proposed criteria.

Melosh \& Marcal (1977) defined an SED-based refinement criteria which was used for mesh enrichment strategies. The differences in the SED from the centroid of the element to other Gauss points were computed and depending on the magnitude of these differences the element was divided into 4 subelements. No strict refinement strategy was thus followed and there is no explicit computation of the terms in (67) thus making it totally distinct from the proposed strategy.

Botkin \& Bennet (1986) treated the variation in strain energy as a measure of the error in the FE solution. Thus, this was also an SED-based estimate and the refinement equation was given by

$$
\left\|V_{e}\right\|_{i} \leq C_{i} \xi_{i}{ }^{k}\left\|D^{k} V\right\|,
$$

where, $\left\|V_{e}\right\|_{i}=$ elemental error in SED, $C_{1}=$ proportionality factor, $\xi_{i}=$ element size measure, $\left\|D^{k} V\right\|=k$-th variation in SED, $k=1$ for linear problems.

Equation (68) is similar in structure to (67). It may be noticed that in (67) both the reference norm and the local error are in terms of SED which is quite different from the proposed strategy where the SED is used to modify only the factor $C_{i}$; the reference norm and the local error terms are in terms of weighted energy and strain energy respectively. 


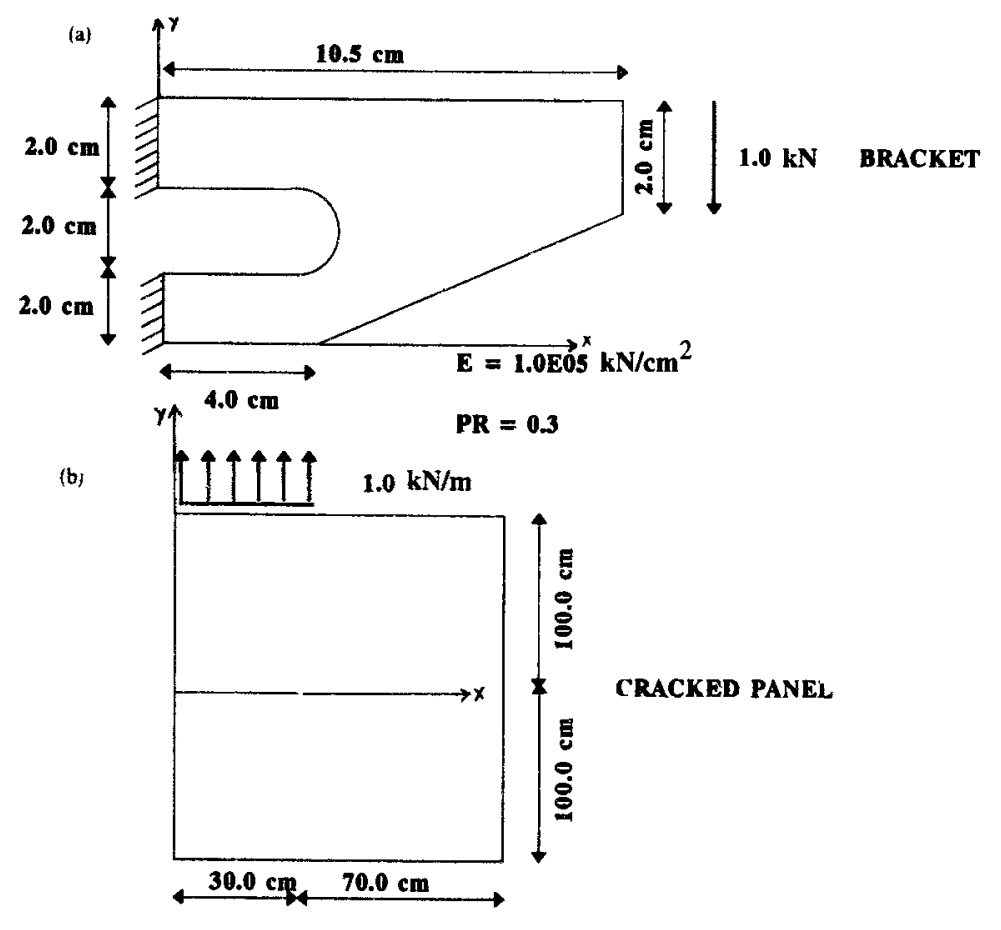

Figure 5. Example problems. (a) Bracket and (b) cracked panel.

The technique proposed by Cedillo \& Bhatti (1988) is again a simple mesh enrichment procedure where a given element is broken into 4 subelements provided the following relationship is valid

$$
\text { LSED } \geq \beta \text { GSED }
$$

where, LSED = local (elemental) strain energy density, GSED = global strain energy density, and $\beta=$ tolerance limit.

In this strategy no explicit error estimation is done and thus no specific refinement strategy can be followed marking its difference from the proposed strategy.

Lee \& Lo (1992) use a SED enhanced scheme as proposed in the current scheme. In Lo's scheme, it is argued that the LSED to GSED ratio is very high for elements near the singularities. To accomodate for this effect, the convergence rate $\lambda$ in (67) is modified by the inclusion of this ratio such that at the presence of singular zones, the convergence rate increases accordingly. Thus, this method is also different from the proposed strategy where the convergence rate is unchanged but the target global error fraction is modified such that it reduces in the presence of a singularity. Lo's algorithm does not modify the reference norm either, as is done in the proposed method.

\section{Case studies}

To demonstrate the efficiency of the proposed refinement scheme over the conventional refinement scheme - two-plane elasticity examples are selected as shown in figure 5, and adaptive FEA is performed with a target global error of $5 \%$. The meshes are shown in 
(a)

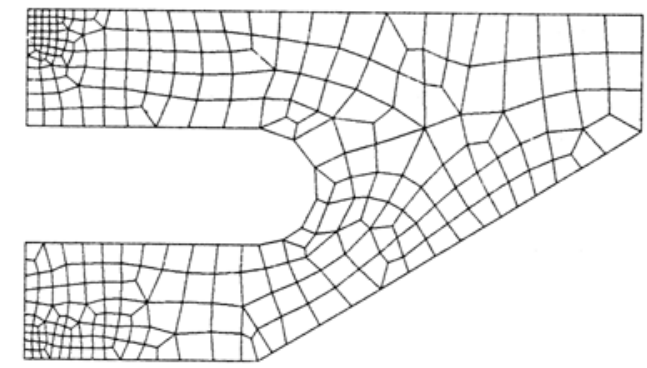

No. of DOF $=746$.

Relative Percent. Error $=9.55$

(b)

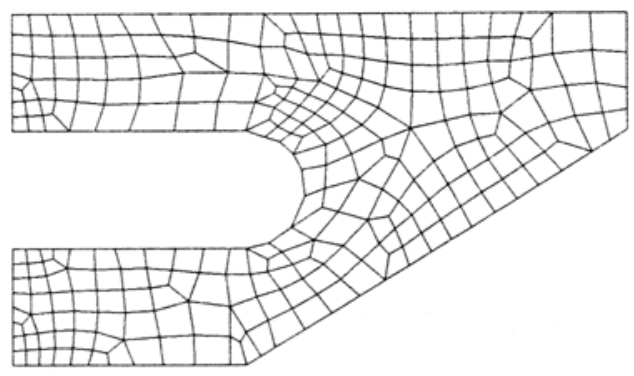

No, of DOF $=572$

Relative Percent Error $=10.94$

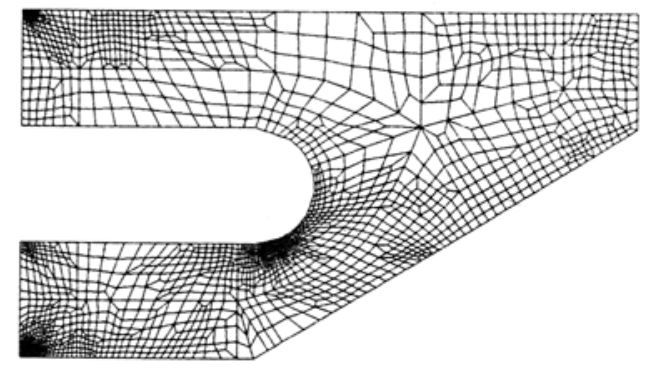

No. of DOF $=3900$

Relative Percent Error $=\mathbf{4 . 7 7}$

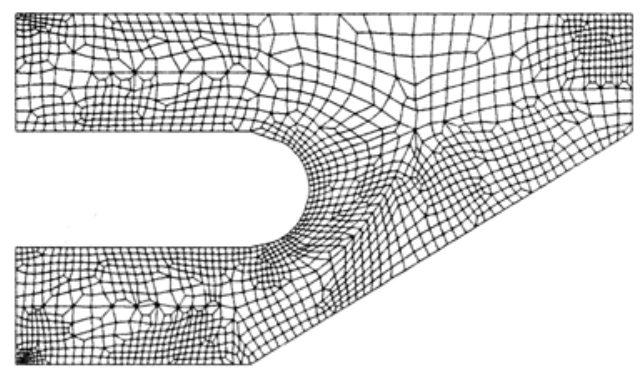

No. of DOF $=3546$

Relative Percent Error $=\mathbf{4 . 7 1}$

Figure 6. Adaptive analysis of a bracket. (a) Conventional and (b) proposed strategies.

figures 6-7, the stress distribution of the cracked panel problem is shown in figure 8 while the convergence plots are shown in figure 9. Of the two problems solved, the bracket is characterized by a complicated geometry and the open crack problem is characterized by a singularity at the crack tip. It is observed that for both the refinement strategies, the non-singular bracket problem yields almost similar results in terms of convergence rates. However, in case of the modified refinement strategy, the mesh shows better localization in the high stress zones. Thus, in non-singular problems, the new refinement strategy brings about a measure of directional refinement - to yield an $r-h$ refinement process. For the bracket problem, the conventional refinement strategy yields a error percentage of 4.77 for a mesh with 3900 degrees of freedom, whereas, using the proposed refinement strategy, 3546 degrees of freedom in the final mesh yield an error percentage of 4.71 . Thus, the proposed strategy yields a more economic solution.

In case of the singular problems, the new refinement strategy yields a better convergence rate in addition to a better localization of the mesh. Thus, in such problems, the $r-h$ method actually shifts nodes closer to the singular zones resulting in higher convergence rates for the same number of degrees of freedom. Using the conventional refinement strategy, 2760 degrees of freedom in the final mesh yield an error percent of 4.72 while the proposed strategy requires only 2524 degrees of freedom to yield an error percent of 4.15 . Thus, 
(a)

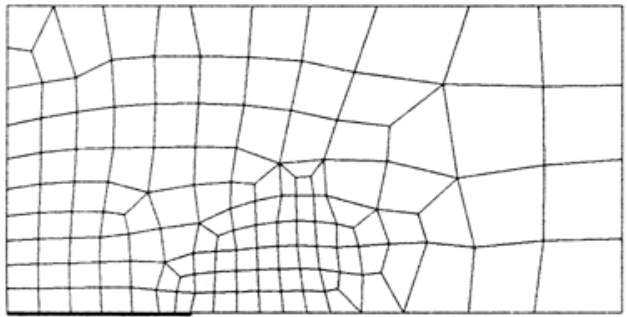

No. of DOF $=260$.

Relative Percent. Error $=\mathbf{1 4 . 4 5}$

(b)

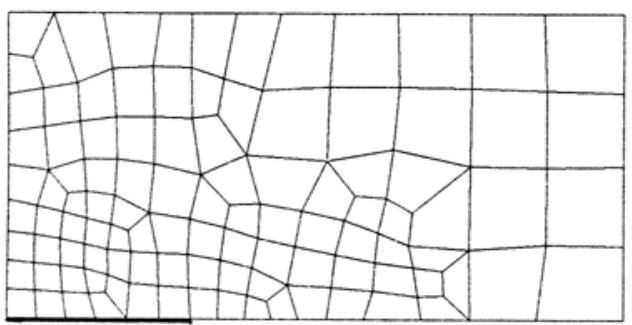

No. of DOF $=226$

Relative Percent. Error $=\mathbf{1 3 . 9 7}$

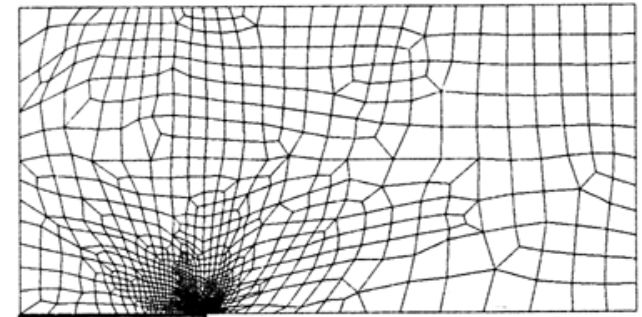

No. of DOF $=2760$.

Relative Percent. Error $\mathbf{=} \mathbf{4 . 7 2}$.

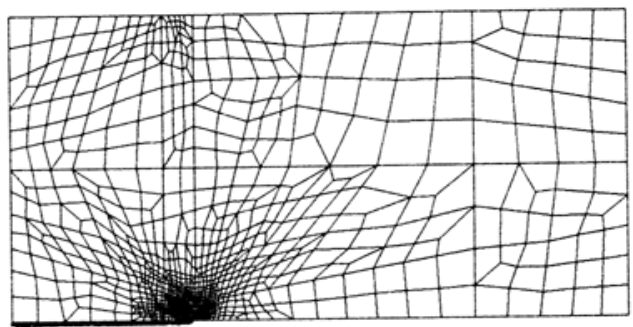

No. of DOF $=2524$

Relative Percent. Error $=4.15$.

Figure 7. Adaptive analysis of a cracked panel. (a) Conventional and (b) proposed strategies.

in the case of this singular problem also, using the proposed strategy results in a more economic solution.

\section{Conclusions}

Based on the work presented here, the following conclusions may be drawn.

(1) The skeleton-based domain decomposition procedure is ideally suited for the decomposition of complex objects into simple mapable subregions.

(2) Since the mesh generator is activated in very simple domains (i.e. either quadrilateral or triangular), the speed of mesh generation is fast.

(3) As each superelement is considered in turn for mesh generation, this method is ideally suited for parallel/distributed computing applications. In sequential computing environments also, substructuring may be adopted easily to bring down computational costs.

(4) The superelement generation process automatically eliminates convex corners from the meshable regions.

(5) The mesh generation process is based on very general splitting procedures, hence this technique may be used in problems with higher dimensions too. 


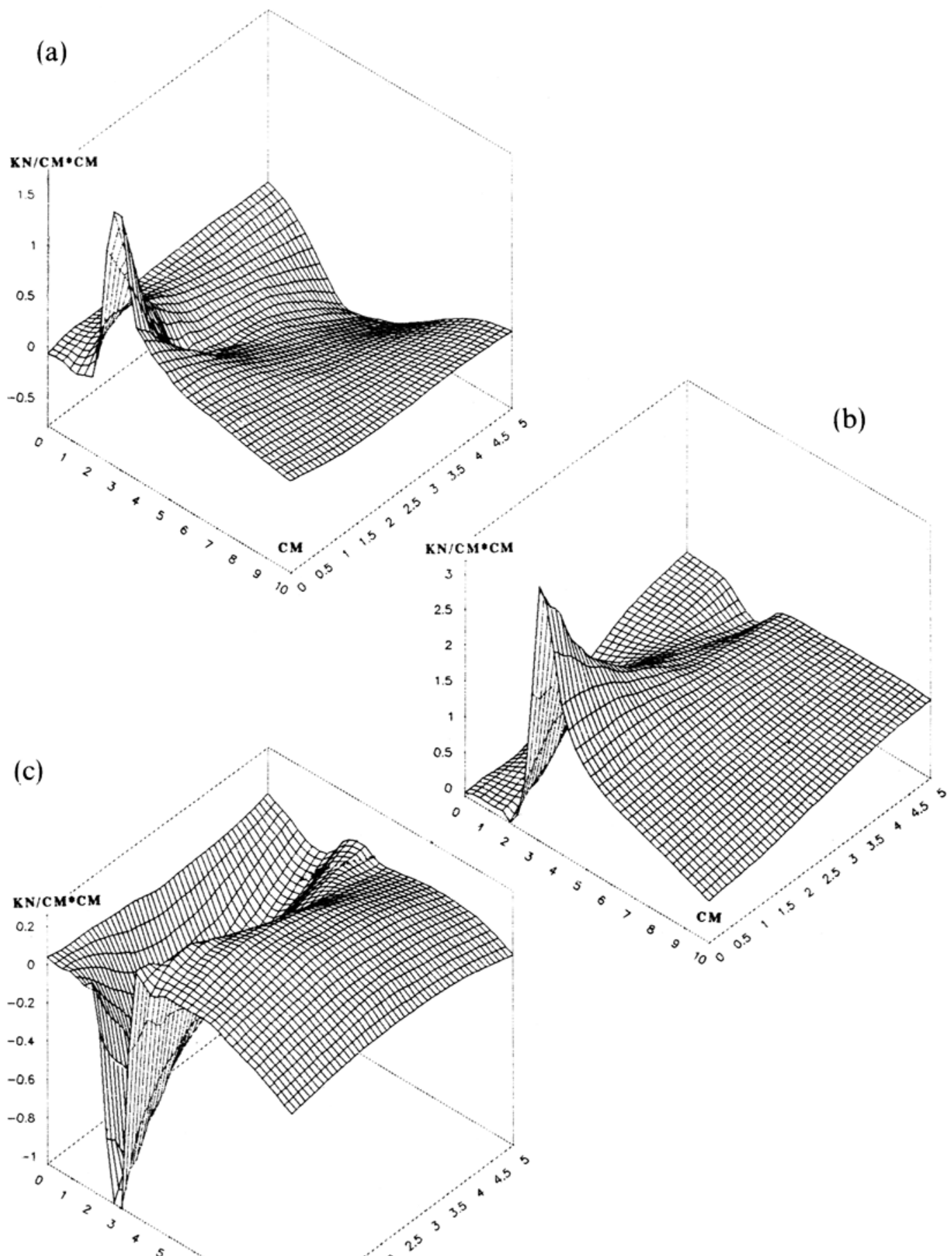

Figure 8. Stress distribution of cracked panel. Variation of $\sigma_{x x}$ (a), $\sigma_{y y}$ (b), and $\sigma_{x y}$ (c). 


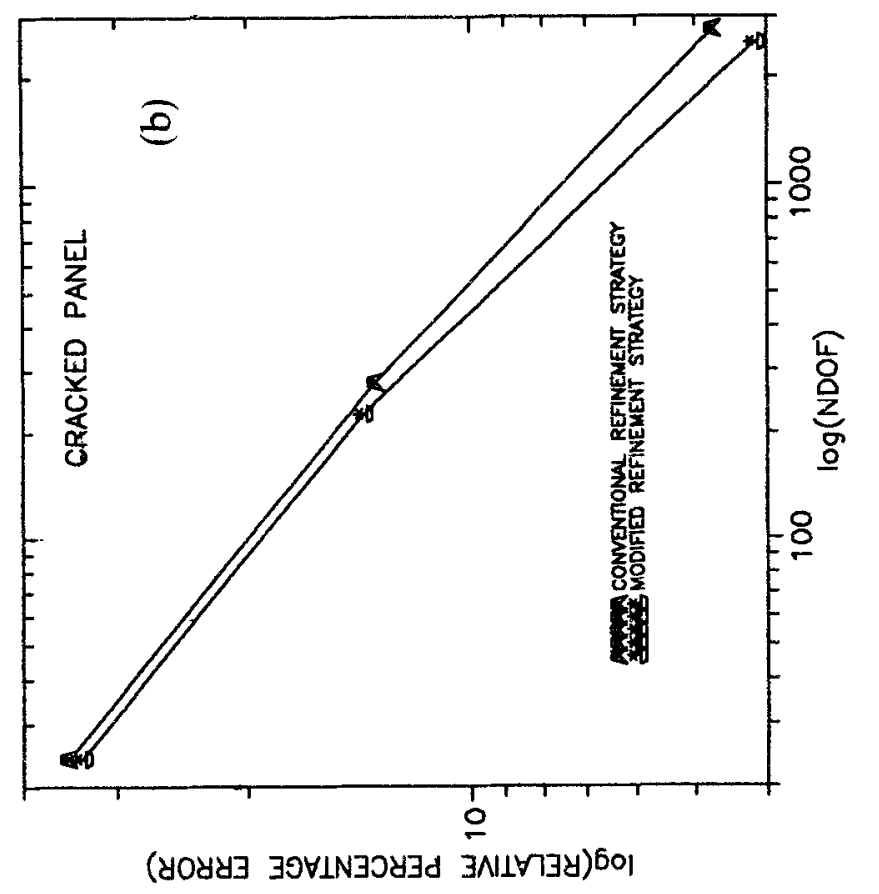

है

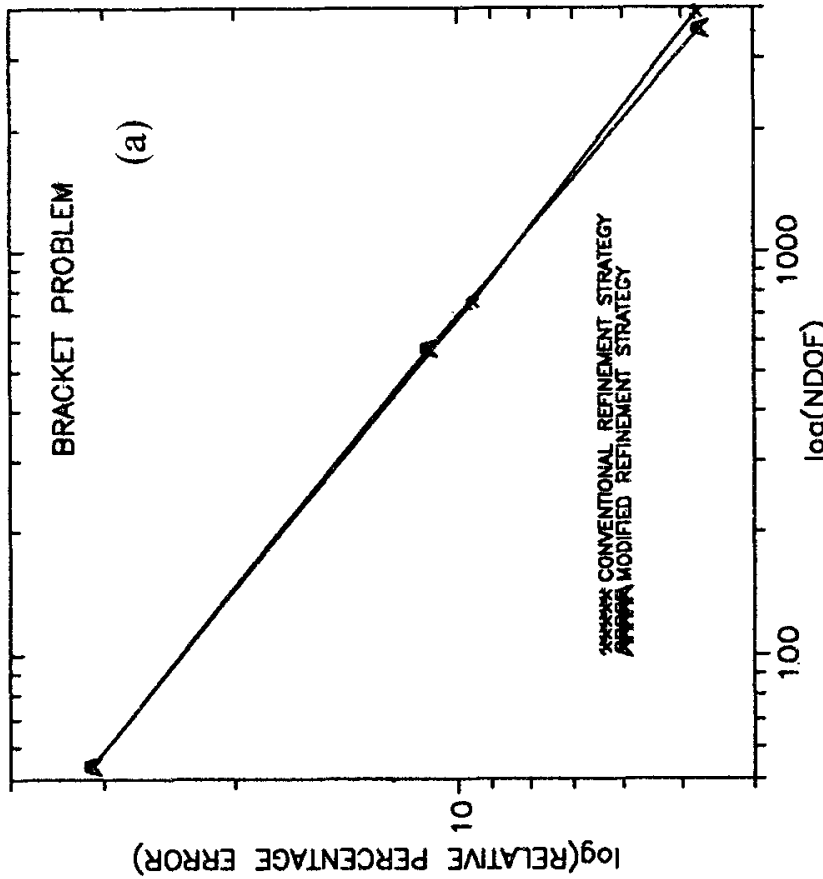


(6) The proposed refinement strategy shows asymptotic rates of convergence, thus dispensing of the equal error distribution paradigm. In fact, it is shown that errors are distributed in proportion to element sizes which automatically imposes more stringent local refinement criteria at high stress zones.

(7) An $r$ - $h$ adaptive method is embedded in an $h$-refinement framework by using the proposed strategy.

(8) In both the problems solved, the modified strategy requires a lesser number of degrees of freedom than conventional strategy to achieve the same global accuracy levels. Thus, the modified refinement strategy makes the adaptive process more economical.

(9) The proposed refinement strategy speeds up convergence for singularity dominated problems thus imparting greater measures of reliability to the FE solutions of such problems.

\section{Future direction of research}

(1) Adaptive analysis of R-M plates using field consistent 4-node elements was reported by the authors (Reddy \& Turkiyyah 1995), where the effects of plate thickness and boundary conditions on adaptivity were discussed.

(2) The skeletal decomposition of general parametric surfaces has been taken up for adaptive analysis of shells.

(3) The mesh generation process is being modified for generation of quadrilateral elements on 4-sided doubly curved superelements.

(4) An optimization based global-local error estimate is being developed for local pointwise error control and asymptotic convergence rates.

(5) A new patchwise superconvergent stress recovery procedure is under development incorporating residuals due to equilibrium violation, natural boundary condition violation and interelement stress differences.

\section{References}

Babuska I, Szabo B 1982 On the rates of convergence of the finite element method. Int. J. Numer. Methods Eng. 20: 323-341

Blacker T, Belytschko T 1994 Superconvergent patch recovery with equilibrium and conjoint interpolant enhancements. Int. J. Numer. Methods Eng. 37: 517-536

Blacker T, Stevenson M 1991 Paving: a new approach to automated quadrilateral mesh generation. Int. J. Numer. Methods Eng. 32: 811-847

Blum H 1967 A transformation for extracting new descriptors of shape. Models for the perception of speech and visual form (ed.) W Wathen-Dunn (Cambridge, MA: MIT Press)

Blum H 1973 Biological shape and visual science. J. Theor: Biol. 38: 205-287

Blum H, Nagel R 1973 Shape description using weighted symmetric axis features. Pattern Recogn. 10: $167-180$

Bookstein F L 1979 The line skeleton. Comput. Graphics Image Process. 11: 123-137 
Botkin M A, Bennet J A 1986 The application of adaptive mesh refinement to shape optimization of plate structures. Accuracy estimates and adaptive refinements in finite element computations (eds) I Babuska, J P Gago, O C Zienkiewicz, D W Keily (New York; John Wiley) pp 227-246

Buell W R, Bush B A 1973 Mesh generation - a survey. J. Eng. Ind., ASME 7: 332-338

Bugeda G, Oliver J 1991 Automatic adaptive remeshing for structural shape optimization. European Conf. on New Advances in Computat. Struct. Mech. Giens, France

Calabi L, Hartnett W E 1968a A theorem for closed non convex sets. Proc. Am. Math. Soc. 19: 1495-1498

Calabi L, Hartnett W E $1968 \mathrm{~b}$ Shape recognition, prairie fires, convex deficiencies and skeletons. Am. Math. Mon. 75: 335-338

Cantin G, Touzot G, Loubignac G 1978 An iterative algorithm to build continuous stress and displacement relations. Int. J. Numer. Methods Eng. 12: 1453-1506

Cedillo H E F, Bhatti M A 1988 A simple strain energy based finite element mesh refinement scheme. Comput. Struct. 28: 523-533

Cook R D 1982 Loubignac's iterative method in finite element elastostatics. Int. J. Numer. Methods Eng. 18: 67-75

Gago J P de S R, Kelly D W, Zienkiewicz O C, Babuska I 1983 A-posteriori error analysis and adaptive processes in the finite element method: Part II - adaptive mesh refinement. Int. J. Numer: Methods Eng. 19: 1621-1656

Grosse I R, Katragadda P, Benoit J 1992 An adaptive accuracy-based a posteriori error estimator. Finite Elements Anal. Design 12: 75-90

Gursoy H N, Patrikalakis N M 1992 An automatic coarse and fine surface mesh generation scheme based on medial axis transform: Part 1. Algorithms. Eng. Comput. 8: 121-137

Heighway E A 1983 A mesh generator for automatically subdividing irregular polygons into quadrilaterals. IEEE Trans. Magnetics 19: 2535-2538

Ho-Le K 1988 Finite element mesh generation methods: A review and classification. Comput. Aided Design. 20: 27-38

Kelly D W 1984 The self equilibration of residuals and complimentary a-posteriori error estimates in FEM. Int. J. Numer. Methods Eng. 20: 1491-1506

Kelly D W, Isles J D 1989 A procedure for a-posteriori error analysis for the FEM which contains a bounding measure. Int. J. Numer. Methods Eng. 31: 63-71

Kelly D W, Gago J P de S R, Zienkiewicz O C, Babuska I 1983 A-posteriori error analysis and adaptive processes in the finite element method: Part I - error analysis. Int. J. Numer. Methods Eng. 19: 1593-1619

Krishnamoorthy C S, Raphael B, Mukherjee S 1995 Meshing by successive superelement decomposition (MSD) - A new approach to quadrilateral mesh generation. Finite Elements Anal. Design 20: 1-37

Lee C K, Lo S H 1992 An automatic adaptive refinement finite element procedure for 2D elastostatic analysis. Int. J. Numer. Methods Eng. 35: 1967-1989

Lee D T 1982 Medial axis transformations of a planar shape. IEEE Trans. Pattern Anal. Mach. Intell. PAMI-4: 363-369

Melosh R J, Marcal P V 1977 An energy basis for mesh refinement of structural continua. Int. J. Numer. Methods Eng. 11: 1083-1091

Montanari U 1968 A method for obtaining skeleton using a quasi-Euclidian distance. J. Assoc. Comput. Mach. 15: 600-624

Mukherjee S, Krishnamoorthy C S 1996 a Adaptive analysis of plates by shear flexible quad4 R-M elements. Finite Elements Anal. Design 22: 329-366

Mukherjee S, Krishnamoorthy C S 1996b Element patch based enhanced superconvergent stress 
projection and adaptive finite element analysis. Int. J. Numer. Methods Eng. (communicated)

Nagel R N, Blum H 1976 A symmetric axis basis for object recognition and description. Proc. IEEE meeting on Decision and Control 168-170

Patrikalakis N M, Gursoy H N 1990 Shape interrogation by medial axis transform. Design Lab Memo, 90-2, Sea Grant College Program, MIT

Reddy J M, Turkiyyah G 1995 Computation of 3D skeletons using a generalized Delaunay triangulation technique. Comput. Aided Design 27: 677-694

Talbert J A, Parkinson AR 1990 Development of an automatic finite element two dimensional mesh generator using quadrilateral elements and Bezier curve boundary definition. Int. J. Numer. Methods Eng. 29: 1551-1567

Tam T K H, Armstrong C G 1991 2D finite element mesh generation by medial axis subdivision. Adv. Eng. Software 13: 313-324

Thacker W C 1980 A brief review of techniques for generating irregular computational grids. Int. J. Numer. Methods Eng. 15: 1335-1342

Turkiyyah G, Fenves S J 1988 Generation and interpretation of finite element models in a knowledge based environment. R-90-188, Department of Civil Engineering, Carnegie-Mellon University

Wiberg N-E, Abdulwahab F 1993 Patch recovery based on superconvergent derivatives and equilibrium. Int. J. Numer. Methods Eng. 36: 2703-2724

Wiberg N-E, Li X D 1994 Superconvergent patch recovery of finite element solution and an a-posterori $L_{2}$ norm error estimate. Commun. Numer. Methods Eng. 10: 313-320

Wiberg N-E, Abdulwahab F, Ziukas S 1994 Enhanced superconvergent patch recovery incorporating equilibrium and boundary conditions. Int. J. Numer. Methods Eng. 37: 3417-3440

Wiberg N-E, Abdulwahab F, Ziukas S 1995 Improved element stress for node and element patches using superconvergent patch recovery. Commun. Numer. Methods Eng. 11: 619-627

Zhu J Z, Zienkiewicz O C, Hinton E, Wu J 1991 A new approach to the development of automatic quadrilateral mesh generation. Int. J. Numer. Methods Eng. 32: 849-866

Zienkiewicz O C, Zhu J Z 1987 A simple error estimator and adaptive procedure for practical engineering analysis. Int. J. Numer. Methods Eng. 24: 337-357

Zienkiewicz O C, Zhu J Z 1992a The superconvergent patch recovery and a-posteriori error estimates. Part 1: The recovery technique. Int. J. Numer. Methods Eng. 33: 1331-1364

Zienkiewicz O C, Zhu J Z 1992b The superconvergent patch recovery and a-posteriori error estimates. Part 2: Error estimates and adaptivity. Int. J. Numer. Methods Eng. 33: 1365-1382 